\title{
High Resolution Electrocardiography Optimised for Recording Pulses from Electronic Pacemakers: Evaluation of a New Pacemaker Sensing System
}

\author{
S Petrutiu ${ }^{1}$, AV Sahakian ${ }^{1}$, A Ricke $^{3}$, B Young ${ }^{3}$, S Swiryn $^{1,2}$ \\ ${ }^{1}$ Northwestern University, Evanston, IL, USA \\ ${ }^{2}$ HeartCare Midwest, Peoria, IL, USA \\ ${ }^{3}$ GE Healthcare, Milwaukee, WI, USA
}

\begin{abstract}
Pacemaker outputs are poorly recorded and displayed by the standard ECG. A new high resolution ECG system optimized for recording outputs from electronic pacemakers was evaluated. Three ECGs with different pulse settings were recorded in 42 patients with pacemakers at a sampling rate of $75 \mathrm{kHz}$ using a new ECG acquisition module. Atrial and ventricular pulses were detected and the measured pulse widths and amplitudes were compared to the programmed values. For the atrium, the correlations between programmed and measured width and amplitude were 0.85 and 0.74 , while for the ventricle they were 0.99 and 0.8. Leads II and VI had the highest atrial amplitude, and leads V3, V4 and V5 had the highest ventricular amplitude. The new high resolution ECG pacemaker system dramatically improved the reproduction of pacemaker outputs, allowing accurate measurement of pulse duration for both atrial and ventricular pulses.
\end{abstract}

\section{Introduction}

The accuracy of computer-based electrocardiogram (ECG) interpretation algorithms has been investigated [1, $2,3,4]$. The most common errors encountered are related to arrhythmias and electronic pacemakers [1,2,3]. Approximately 5\% of all diagnostic ECGs are acquired in patients with implanted electronic pacemakers [5]. The number of patients with pacemakers is growing due to an aging population and the pacemaker technology is evolving; therefore improvements in automated ECG interpretation algorithms should be focused on this area.

Within the group of patients with pacemakers, the most frequent error is the failure of the computer to identify the presence of a pacemaker $[2,4]$. This is due to pacemaker outputs being poorly recorded and displayed. Most pacemakers use bipolar electrodes which generate much smaller voltages on the surface ECG when compared to unipolar electrodes [6]. Pulse durations are short resulting in very narrow pulses. A common error of automated ECG interpretation algorithms is completely missing the ventricular pulses or both atrial and ventricular pulses. This then cascades into other misinterpretations such as conduction defects or infarction. An improved software-based pacemaker pulse detection algorithm has been proposed [5].

The diagnosis of certain arrhythmias including atrial fibrillation is often based on the presence of an irregular ventricular rhythm. The absence of an irregular rhythm in ECGs from patients with pacemakers results in underrecognition of the underlying $\mathrm{AF}$ and therefore leads to under-treatment [7].

As the 12-lead surface ECG continues to aid pacemaker implantation follow-up for pacemaker recipients, the goal of paced rhythm analysis systems is to accurately detect and classify pacemaker pulses as well as properly display them to physicians for correct diagnosis and further investigation. A new high-resolution 12-lead ECG system has been developed (GE Healthcare, Milwaukee, WI) [8]. In this study this new highresolution system optimized for recording outputs from electronic pacemakers was evaluated. This new system will allow improvements in both human and computer diagnostic and interpretation algorithms for patients with electronic pacemakers.

\section{Methods}

\subsection{Recordings}

Three high resolution 12-lead ECGs using a new ECG acquisition module (GE Medical, Milwaukee, WI) as well as standard 12-lead ECGs were recorded in each patient. The sampling rate for each high resolution ECG was $75000 \mathrm{~Hz}$. The duration of each ECG was 12 seconds. Different pulse settings were programmed for each ECG. The programmed pulse duration and amplitude were reduced between the first and the second ECG and between the second and the third ECG. 


\subsection{Data processing}

Digital signal processing was performed using MATLAB (The Mathworks Inc., Natick, MA). Automated atrial and ventricular pacemaker pulse detection was performed for each high resolution ECG in each patient. A three-point central difference differentiation algorithm was used to detect the leading and trailing edges of each pacemaker pulse in each lead. For each detected pacing pulse, the pulse width and amplitude were computed.

Figure 1 shows an example of lead V1 from a high resolution ECG from a patient with a dual-chamber pacemaker. Atrial pulses are marked by black dots and ventricular pulses are marked by red dots.

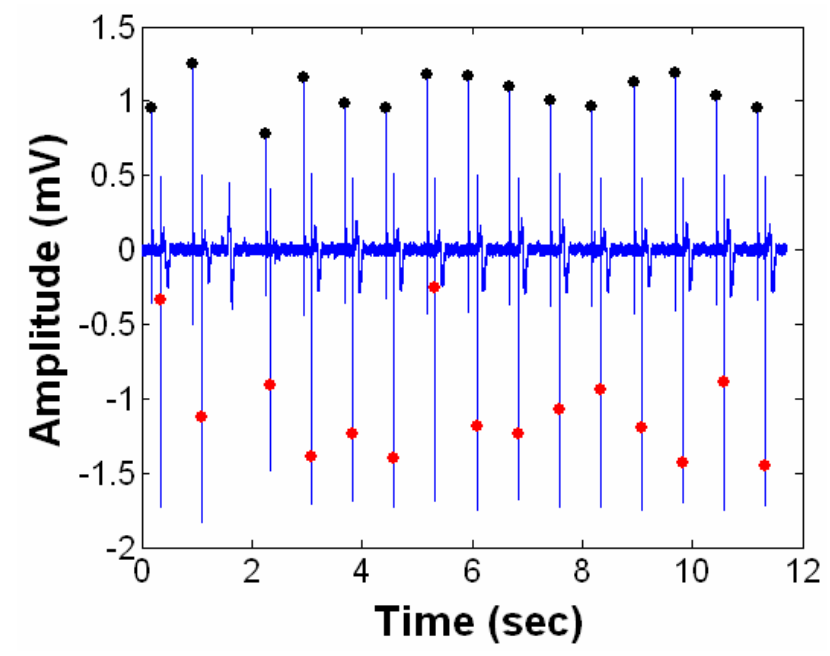

Figure 1: Atrial and ventricular pulse detection from lead V1 of a high resolution ECG; atrial pulses are marked by black dots and ventricular pulses by red dots.

Figures 2 and 3 show an example of an atrial pulse with the pulse amplitude and pulse width marked by black dots. Figures 4 and 5 show an example of a ventricular pulse with the pulse amplitude and pulse width marked by red dots.

For each lead, the median pulse width and amplitude were computed. The overall median measured pulse width was compared to the programmed pulse width.

To evaluate the pulse amplitude, the ratio of programmed pulse amplitude between the first and second ECG and between the second and third ECG was calculated. This ratio was compared with the ratio of the measured pulse amplitudes from the corresponding ECGs.

To determine which leads are optimal for recording atrial versus ventricular pulses, the ECG leads with the highest atrial and ventricular pulse amplitude were identified in each patient. For the patients with the same programmed atrial and ventricular amplitude, the measured atrial pulse amplitude was compared to the measured ventricular pulse amplitude.

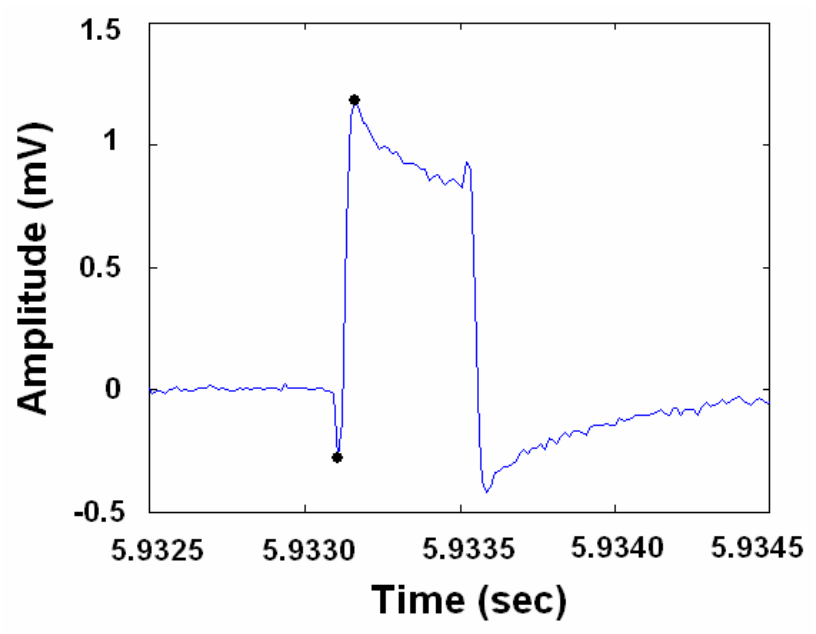

Figure 2: Atrial pulse amplitude

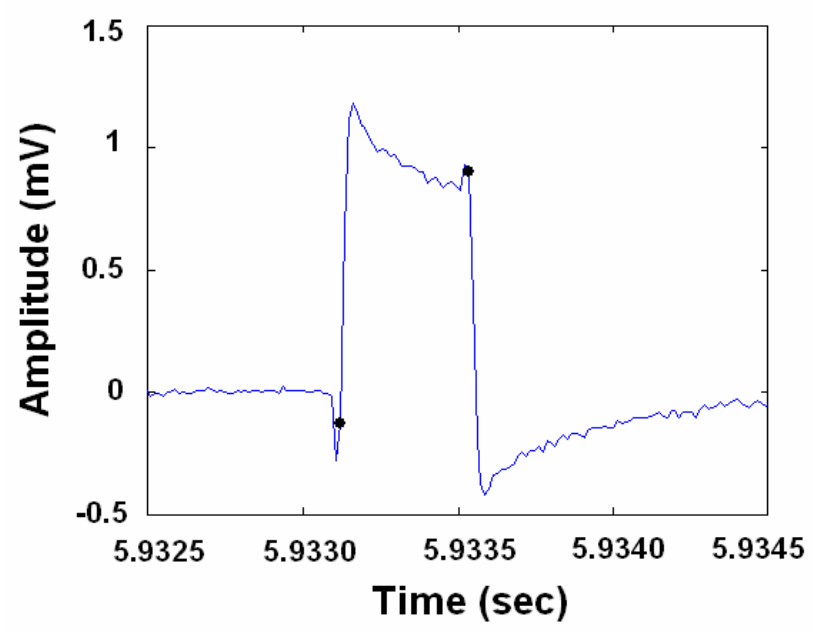

Figure 3: Atrial pulse width

\section{Results}

A total of 42 patients with a variety of pacemakers including 27 dual-chamber pacemakers were included in this study. There were 32 male and 10 female and their age ranged from 32 to 86 .

There was a striking improvement in the display of pacemaker outputs for the $75000 \mathrm{~Hz}$ data compared to standard ECG recordings. Table 1 shows the ranges for the programmed pacemaker pulse widths and amplitudes. For both atrium and ventricle, the programmed pulse durations ranged from 0.4 to $0.6 \mathrm{~ms}, 0.2$ to $0.3 \mathrm{~ms}$, and 
0.03 to $0.05 \mathrm{~ms}$ for the first, second and third ECG, respectively. Programmed pulse amplitudes ranged from 2.0 to $5.5 \mathrm{~V}, 1$ to $2.5 \mathrm{~V}$, and 0.25 to $0.5 \mathrm{~V}$, respectively.

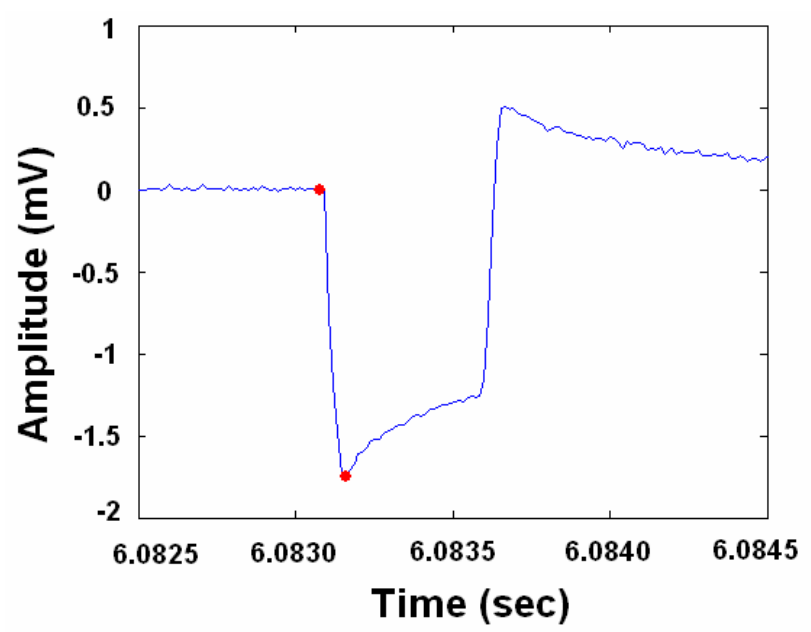

Figure 4: Ventricular pulse amplitude

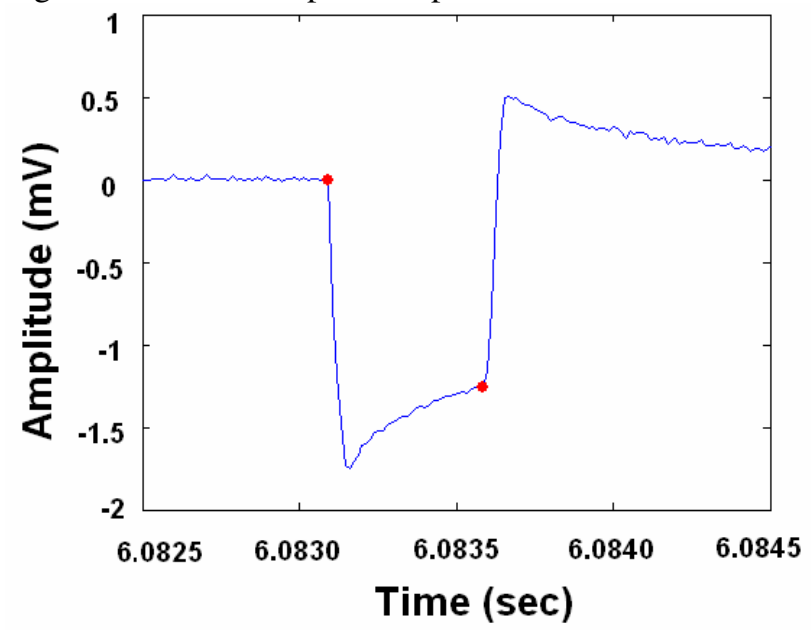

Figure 5: Ventricular pulse width

\begin{tabular}{|c|c|c|c|}
\hline & ECG 1 & ECG 2 & ECG 3 \\
\hline $\begin{array}{c}\text { Programmed } \\
\text { Pulse } \\
\text { Width (ms) }\end{array}$ & $0.4-0.6$ & $0.2-0.3$ & $0.03-0.05$ \\
\hline $\begin{array}{c}\text { Programmed } \\
\text { Pulse Amplitude } \\
(V)\end{array}$ & $2.2-5.5$ & $1.0-2.5$ & $0.25-0.5$ \\
\hline
\end{tabular}

Table 1: Ranges of programmed atrial and ventricular pulse widths and amplitudes for each of the three ECGs

Table 2 shows the correlations between programmed and measured values for both atrium and ventricle. For the atrium, the correlation between the programmed and the measured duration was 0.85 . The correlation between changes in programmed and measured amplitude was 0.74. For the ventricle, the correlation between the programmed and the measured duration was 0.99 . The correlation between changes in programmed and measured amplitude was 0.8 .

\begin{tabular}{|c|c|c|}
\hline & Correlation & p-value \\
\hline Atrial pulse width & 0.85 & 0.0001 \\
\hline Atrial pulse amplitude & 0.74 & 0.0001 \\
\hline Ventricular pulse width & 0.99 & 0.0001 \\
\hline Ventricular pulse amplitude & 0.80 & 0.0001 \\
\hline
\end{tabular}

Table 2: Correlations between programmed and measured values for both atrium and ventricle

For the atrium, leads II and V1 had the highest pulse amplitude in 16 out of 27 cases. For the ventricle, leads V3, V4 and V5 had the highest pulse amplitude in 33 out of 41 patients with ventricular pacing, with V4 showing the highest amplitude in most cases. The variability was greater for atrial pulses compared to ventricular pulses. For the 15 patients with the same programmed atrial and ventricular pulse amplitude, the measured median lead ventricular amplitude was greater than the atrial amplitude in 12 out of 15 patients.

\section{Discussion and conclusions}

A number of studies have investigated the accuracy of automated 12-lead ECG interpretation algorithms [1, 2, 3,4]. It has been shown that automatic ECG interpretation from patients with implanted electronic pacemakers often needs revision by cardiologists. A study by Guglin et al. that focused only on the group of patients with pacemakers showed that the computer-based interpretation of $61.3 \%$ of ECGs with electronic pacemakers required revision [4], while a study by Poon et al. showed that $75.2 \%$ from this group required revision [3].

The most common error involving the interpretation of ECGs with pacemakers is the failure to identify the presence of a pacemaker [2,4]. The presence of a pacemaker was missed in $10.2 \%$ of cases in one study [3] and in $18.4 \%$ of the cases in another [4].

Dual-chamber pacing is often misclassified as just ventricular pacing [4]. Other common errors include under-recognition of the underlying rhythm, such as sinus rhythm with dual chamber pacing [3] or atrial fibrillation $[4,7]$. This can result in under-treatment and an increased risk of additional complication such as cerebrovascular accidents in the case of atrial fibrillation [7]. An improved software-based pacemaker pulse 
detection algorithm has been proposed [5]. However, the display of pacemaker pulses was not improved.

The new high resolution ECG pacemaker system dramatically improved the reproduction of pacemaker outputs. This system allowed the accurate measurement of pulse width and relative pulse amplitude for both atrial and ventricular pulses. Different ECG leads were found to be optimal for recordings pacing outputs for the atrium compared the ventricle. This new system will allow improvements in both human and automated ECG interpretation.

\section{Acknowledgements}

We gratefully acknowledge the contributions of Chris Jordan R.N., Pooja Mehta and Jason Chin to this project. This work was supported in part by a grant from the Dr. Scholl foundation.

\section{References}

[1] Swiryn S, Jenkins J. Arrhythmia diagnosis by computer: Marquette 12SL program. Computerized interpretation of ECG. Proc. Eng. Foundation Conf, 1983;35-54.

[2] Guglin ME, Datwani ND. Common errors in computer electrocardiogram interpretation. International $\mathbf{J}$ of Cardiology 2006; 106:232-7
[3] Poon K, Okin PM, Kligfield P. Diagnostic performance of a computer-based ECG rhythm algorithm. J of Electrocardiology 2005; 38:235-8

[4] Guglin ME, Datwani ND. Electrocardiograms with pacemakers: accuracy of computer reading. $\mathrm{J}$ of Electrocardiology 2007; 40:144-6

[5] Helfenbein ED, Lindauer JM, Zhou SH, Gregg RE, Herleikson EC. A software-based pacemaker pulse detection and paced rhythm classification algorithm. $\mathbf{J}$ of Electrocardiology 2002; 35;95-103

[6] Ellenbogen KA, Wood MA. Cardiac pacing and ICDs: Third edition, Massachusetts; Blackwell Science 2002

[7] Patel AM, Westveer DC, Man C, Stewart JR, Frumin HI. Treatment of underlying atrial fibrillation: paced rhythm obscures recognition. J of American College of Cardiology 2000; 36;784-7

[8] Ricke A, Haisty W, Kroeger S, Swiryn S, Young B. Advanced pacemaker detection. ISCE Abstract 2007, in press.

Address for correspondence

Alan V Sahakian

Northwestern University

Departments of EECS and BME

2145 Sheridan Rd

Evanston, IL 60208-3118, USA

sahakian@ece.northwestern.edu 